\title{
openheart Impact of ultrasound contrast agent on the detection of thrombi during transoesophageal echocardiography
}

\author{
Henning Ebelt, Alexandra Offhaus, Matthias Wiora, Peter Roehl, \\ Andreas Schwenzky, Anja Weida, Matthias Hoyme, Juliane Bindemann-Koecher, \\ Jelena Anacker
}

\begin{abstract}
- Additional material is published online only. To view please visit the journal online (http://dx.doi.org/10.1136/ openhrt-2019-001024).
\end{abstract}

To cite: Ebelt $\mathrm{H}, \mathrm{Offhaus} \mathrm{A}$, Wiora M, et al. Impact of ultrasound contrast agent on the detection of thrombi during transoesophageal echocardiography. Open Heart 2019;6:e001024. doi:10.1136/ openhrt-2019-001024

Received 31 January 2019 Revised 14 July 2019 Accepted 12 September 2019
Check for updates

(c) Author(s) (or their employer(s)) 2019. Re-use permitted under CC BY-NC. No commercial re-use. See rights and permissions. Published by BMJ.

Department of Medicine II, Catholic Hospital 'St. Johann Nepomuk', Erfurt, Germany

Correspondence to Dr Henning Ebelt; henningebelt@gmail.com

\section{ABSTRACT}

Background Atrial fibrillation (AF) carries the risk of thrombus formation in the left atrium and especially in the left atrial appendage (LAA). A transoesophageal echocardiography (TOE) is routinely performed in these patients to rule out thrombi before cardioversion or structural interventions like LAA closure or pulmonary vein isolation. However, in a certain number of cases, inconclusive results of the TOE may result. This study was performed to analyse whether the routine use of ultrasound contrast agent (UCA) has an influence on the frequency of thrombus detection.

Methods In patients with AF who were scheduled for a subsequent interventional procedure, a TOE was initially performed without contrast agent. Then, the TOE was repeated with the use of UCA. The percentage of diagnostic findings regarding the prevalence of thrombus in the LAA with and without UCA were compared (thrombus present $(\mathrm{T}+)$, no thrombus ( $\mathrm{T}-$ ) and inconclusive result $(\mathrm{T}+/-))$.

Results 223 patients were prospectively included into the trial. The numbers of thrombus detection were as follows: without UCA: $17 \mathrm{~T}+(7.6 \%), 154 \mathrm{~T}-(69.1 \%), 52$ $\mathrm{T}+/-(23,3 \%)$; with UCA: $16 \mathrm{~T}+(7.2 \%), 179 \mathrm{~T}-(80.3 \%)$, $28 \mathrm{~T}+/-\left(12.6 \% ; \chi^{2}: p<0.01\right)$. In 29 examinations $(13.0 \%)$, the use of UCA had an impact on the subsequent treatment strategy.

Conclusions The use of UCA during TOE in patients with AF has a significant impact on the subsequent patient management especially due to an improved rule out of LAA thrombi.

\section{INTRODUCTION}

Atrial fibrillation (AF) occurs in approximately $0.4 \%-1 \%$ of the general population and is increasing with age to $>8 \%$ in those $>80$ years of age ${ }^{1-3}$ As AF is closely linked to the formation of thrombi in the left atrium and especially in the left atrial appendage (LAA), it is of utmost importance to minimise the risk for cardioembolic complications especially before elective procedures like cardioversion $(\mathrm{CV})$, pulmonary vein isolation $(\mathrm{PVI})$ or LAA closure (LAAC). The standard of care to identify LAA thrombi is transoesophageal

\section{Key questions}

What is already known about this subject?

- In previous studies, it has been reported that the use of ultrasound contrast agent (UCA) may improve the diagnostic yield of transoesophageal echocardiography regarding the detection of thrombi in patients with atrial fibrillation (AF). However, these studies were rather limited in sample size and did not analyse whether the use of the UCA would finally translate into a change in the subsequent treatment of the patients.

What does this study add?

- This study shows that the routine use of UCA in patients with AF changes the diagnosis regarding the detection of thrombi in $17 \%$, which is mostly based on an improved rule out of thrombus in otherwise inconclusive situations. This translates into a change in the subsequent treatment strategy in $13 \%$ of these patients mainly due to a reduction of the (unnecessary) postponing of subsequent procedures.

How might this impact on clinical practice?

- The standardised use of UCA might significantly reduce the inappropriate deferral of scheduled procedures in patients with AF.

echocardiography (TOE), which is a semi-invasive procedure that is widely available and provides real-time assessment of cardiac structures with a high spatial and temporal resolution. Other imaging techniques that could be used alternatively are cardiac CT or cardiac MRI, respectively, but these are currently used in a limited number of situations only (review in: ref $^{4}$ ).

Despite the wide application of TOE in the management of patients with $\mathrm{AF}$, it has been described that in clinical practice there is a rather high percentage of inconclusive TOE results. Likewise, von der Recke and colleagues ${ }^{5}$ reported inconclusive TOE findings in 41 out of 121 patients $(34 \%)$ with $\mathrm{AF}$ who were scheduled for CV. 
There are a number of commercially available intravenous ultrasound contrast agents (UCA), which are capable to pass the pulmonary circulation and to reach systemic circulation (review in: ref 6). Besides their application in the sonography of abdominal organs like the liver, these agents also can be used to opacify the left heart chambers. This allows an enhanced identification of endocardial borders especially during stress echocardiography and identification of intracardiac masses. ${ }^{7}$ So far, only a limited number of studies have investigated the potential role of UCA in patients with AF to detect or exclude thrombi in the LAA. ${ }^{5-10}$ However, these studies reported an improvement of the diagnostic yield of the performed TOE when UCA was used especially due to a reduction in artefacts and suppression of dense spontaneous echo contrast. ${ }^{11}$

Within the last years, the spectrum of routinely performed elective therapeutic interventions in patients with $\mathrm{AF}$ has been widened from $\mathrm{CV}$ to catheter ablation and LAAC. Therefore, there is an increasing need to safely identify and exclude thrombi in the LAA in such patients in order to both reduce the risk of thromboembolic events resulting from a mobilisation of thrombi during these elective interventions as well as to avoid the postponing of scheduled procedures due to an uncertainty of thrombus exclusion. This study was performed to analyse whether the use of UCA in daily practice can increase the diagnostic yield of TOE in this regard.

\section{METHODS}

A prospective single-centre trial was performed between 1 January 2017 and 1 April 2018 ('Impact of the use of ultrasound contrast agent on the detection of thrombi in the left atrial appendage during transesophageal echocardiography (CONDOR)', registered at German Registry of Clinical Trials, DRKS00011716). Adult patients $(\geq 18$ years) with non-valvular AF (paroxysmal, persistent or permanent) with a clinical indication for TOE due to a planned intervention (CV, LAAC or ablation) were eligible if they had no contraindication regarding TOE nor the UCA and if they had no history of previous LAAC (neither interventional nor surgical; for detailed list of inclusion and exclusion criteria, see online supplementary table 1).

TOE was performed according to clinical standards using a General Electrics Vivid E9 equipped with a 3D probe $(6 \mathrm{~T})$. Patients received local anaesthesia of the throat with $2 \%$ xylocaine pump spray (Aspen Germany $\mathrm{GmbH}$, Munich, Germany) and afterwards 2-5mg midazolame intravenous (Ratiopharm, Ulm, Germany). LAA was first examined without the use of UCA at $0^{\circ}$, $45^{\circ}, 90^{\circ}$ and $135^{\circ}$ at imaging settings used in clinical routine (harmonic imaging, frequency $5.0 \mathrm{MHz}, 63.1$ frames per second), and the results were documented into a standardised form to report whether all the views could be obtained, whether a thrombus was present (yes/ no/uncertain) and whether the subsequently planned intervention (CV/LAAC/ablation) could be performed or not (evaluations based on the clinical decision of the performing cardiologist). After completion of this evaluation, the settings of the echo machine were adjusted for the use of UCA by reducing accoustic power at $-32 \mathrm{db}$ to $-28 \mathrm{db}$ resulting in a mechanical index of 0.04-0.1 (all other settings left unchanged). Afterwards $1.0 \mathrm{~mL}$ of the UCA Sonovue (Bracco International B.V., Amsterdam, The Netherlands) was administered through an antecubital vein followed by a bolus of $10 \mathrm{~mL}$ saline. The LAA was again examined at $0^{\circ}, 45^{\circ}, 90^{\circ}$ and $135^{\circ}$, and all evaluations mentioned above were repeated and documented on the standardised form. If the image quality after the administration of UCA was not satisfying in the perspective of the operator, a second bolus of $1.0 \mathrm{~mL}$ SonoVue could be given. At this time, the results of the first measurements were known to the operator.

\section{Statistical analysis}

Statistical data analysis was performed using SPSS Statistics (V.25.0). Differences in frequency of nominally scaled variables were compared by means of Pearson's $\chi^{2}$ test. The McNemar test or the Stuart-Maxwell Test for marginal homogeneity were used in online supplementary tables 3-6, respectively. Metric variables are expressed as mean $\pm \mathrm{SD}$. The basis for the test decisions was a significance level of $\mathrm{p}<0.05$.

\section{RESULTS}

Two hundred and twenty-three patients were consecutively included into the prospective CONDOR study (Impact of the use of ultrasound contrast agent on the detection of thrombi in the left atrial appendage during transesophageal echocardiography), patient characteristics are given in online supplementary table 2. In all patients, the TOE was performed successfully, and no patient did show any side effect of the UCA. The results regarding thrombus detection are given in online supplementary table 3. Examples of three individual patients with typical findings are given in online supplementary figure 1.

As seen from online supplementary table 3 and figure 1 , the administration of UCA had a significant impact on the detection of thrombi in the LAA. As the main finding, in 29 patients, the use of UCA led to definitive diagnosis in otherwise inconclusive situations meaning a rule out of LAA thrombi in 27 cases and the diagnosis of thrombus in two cases (online supplementary table 4). Additionally, in five patients the diagnosis of 'thrombus present' and in another four patients the diagnosis 'no thrombus' was changed after the use of UCA, respectively.

As a consequence of the changed diagnosis of thrombus detection, UCA administration also had a significant impact on the clinical judgement whether the therapeutic procedures that were planned subsequently could be performed. As seen in detail from online supplementary tables 5 and 6 , UCA led to a change in patient 


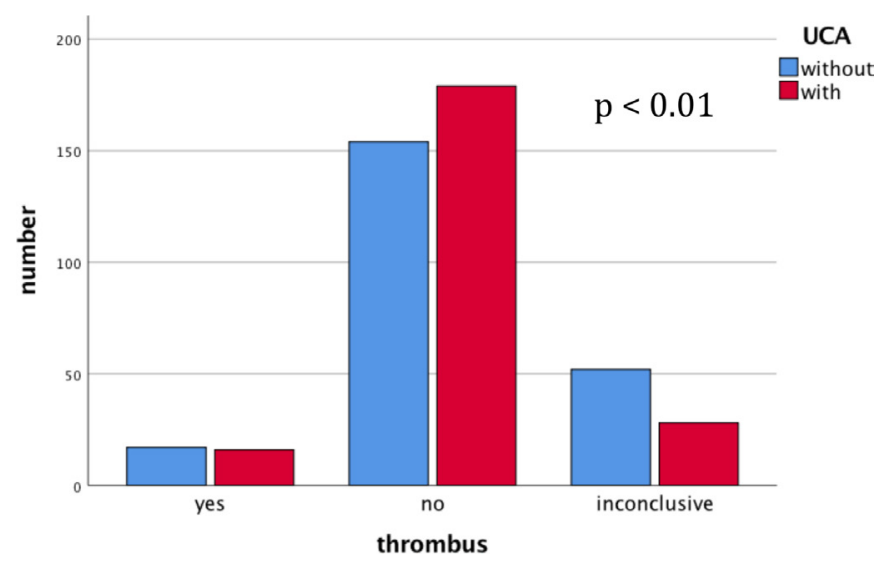

Figure 1 Frequency of thrombi found during TOE examination depending on the administration of ultrasound contrast agent (UCA). TOE, transoesophageal echocardiography.

management in 29 cases with the main effect arising from a $38 \%$ reduction in postponing of scheduled procedures (figure 2).

Data regarding clinical outcomes of the study patients are given in online supplementary table 7 . A percentage of 74.9 of the patients were finally treated with either PVI, LAAC or CV, respectively.

During follow-up until hospital discharge, there was no death, stroke or systemic embolism occurring in any study patient, but one transitoric ischaemic attack was diagnosed in a patient 2 days after electrocardioversion. However, it is noteworthy that in this patient no thrombus was detected during TOE before CV neither without nor with UCA.

\section{DISCUSSION}

In clinical practice, TOE is the standard method to search for intracardiac thrombi in patients with $\mathrm{AF}$. TOE is routinely done before therapeutic procedures like CV or ablation and has to be performed especially if the

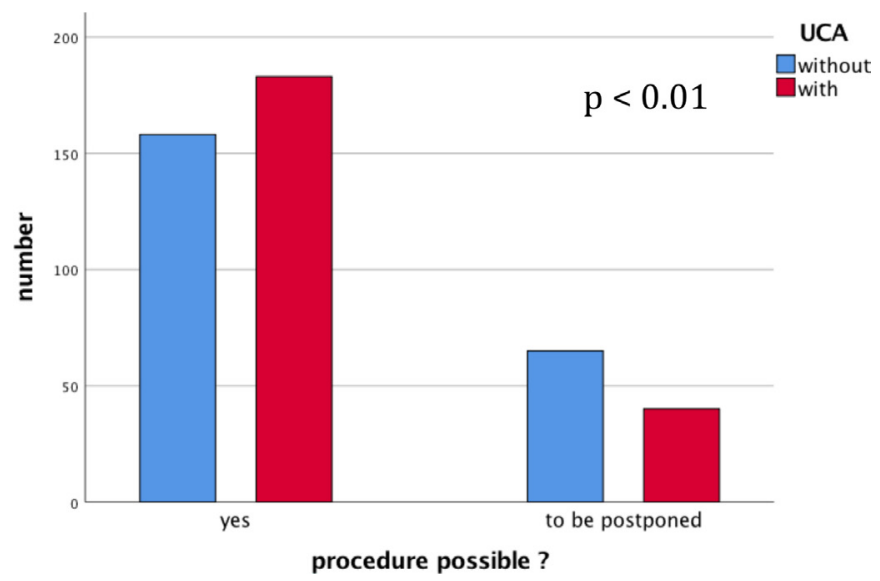

Figure 2 Frequency of the advice to perform or to postpone a subsequently planned procedure according to the TOE result with or without the use of ultrasound contrast agent (UCA). TOE, transoesophageal echocardiography. patient has not been under sufficient anticoagulation for at least the preceding 21 days. ${ }^{12}$ It is of utmost importance to safely rule out the presence of thrombi as the vast majority of such procedures are done in an elective manner so that the avoidance of stroke or systemic embolism is of crucial importance. Hence, if the existence of thrombus cannot be ruled out completely and any doubt is remaining, the responsible echocardiographist very often will advise to postpone any procedure that otherwise could put the patient at risk. Indeed, retrospective analysis of TOE, which had been performed at our institution in patients with AF to rule out thrombi between June 2015 and June 2016 (before UCA was introduced into clinical praxis), showed that out of 202 TOE examinations, LAA thrombi could be confidently ruled out in $59 \%$ (119 exams) and were present in 11\% (22 exams), while inconclusive results were obtained in as much as $30 \%$ (61 exams), respectively. This is in line with previously published reports like a study from von der Recke et $a l,{ }^{13}$ who described $34 \%$ inconclusive TOE results in a cohort of patients who were scheduled for CV (41 out of 121 patients).

In the literature, there are a limited number of reports that showed that the use of UCA can increase the diagnostic accurancy of TOE in patients with $\mathrm{AF}^{8-10} 1314$ However, the numbers of patients included in prospective studies reported so far were rather limited, and there are no data on the influence of the incremental information arising from the administration of UCA on the subsequent patient management. As the number of invasive procedures in patients with $\mathrm{AF}$ has increased substantially in the past few years, it is of high importance to avoid a postponing of interventions related to the uncertainty of diagnostic findings during TOE. also in order to use cathlab capacities and related resources effectively. Hence, in our study, we show that the use of UCA reduces the inappropriate deferral of scheduled procedures by $38 \%$ (25 out of 65 ). Taking into consideration that the costs of the UCA in our study were approximately $€ 30$ per examination ( $€ 105.91$ per $5 \mathrm{~mL}$ of UCA) and the TOE examinations were prolonged only for a few minutes by the application of the contrast agent, it seems to be reasonable to implement the administration of UCA in patients with AF who are scheduled for an interventional procedure. It is important to note that we did not observe any adverse event arising from the contrast agent, which is in line with the known safety profile of the substance.

It was our intention to design this study in correspondence with the clinical situation where an echocardiographist would implement the information of UCA administration after the completion of the 'native' TOE. Therefore, in all TOE examinations, the result regarding thrombi and the possibility of the subsequently planned procedures was first documented without UCA before the contrast agent was applied and the same investigator repeated the evaluation. This also leads to the situation that in our study the incremental effect of UCA can 
be determined in a case-based manner for every single patient, unlike in a study design where independent groups of patients are compared who either received UCA or not, which makes it impossible to determine the direct effect of the UCA on the diagnosis on an individual basis. $^{8}$

Taken together, our data show that the administration of UCA increases the diagnostic yield of TOE in patients with $\mathrm{AF}$ and avoids the unnecessary postponing of subsequent procedures. It can be assumed that the strength of the UCA especially lies in cases where sludge or artefacts make it difficult to securely exclude the occurrence of thrombi in the 'native' TOE.

\section{Limitations}

The study was conducted on an observational basis without blinded evaluation of the echo images. Therefore, an observer bias may be present although this rather reflects the real-world situation in clinical practice. Additionally, there was no anatomical 'gold standard' to confirm the prevalence of a thrombus, as could be provided in case of subsequent cardiac surgery and direct visual validation. The estimated rate for cardioembolic events after cardioversion in anticoagulated patients in the literature is about $1 \%$ and was even lower in our study $(0.4 \%)$. On the basis of this low event rate, no statistical evaluation can be given whether the implementation of UCA has an impact on the frequency of thromboembolic events.

\section{CONCLUSIONS}

Our study shows that in patients with AF, the application of a UCA during TOE is feasible and safe and leads to a changed diagnosis regarding the prevalence of thrombi in a significant proportion of cases. As the main finding, UCA reduces the percentage of otherwise inconclusive TOE findings and avoids the unnecessary postponing of scheduled interventions in these patients.

Contributors All authors have contributed to the final manuscript. HE: planning and conduct of the study, writing of the manuscript and responsible for the overall content. A0: planning and conduct of the study and manuscript revision. MW, PR, $\mathrm{AS}, \mathrm{JB}-\mathrm{K}$ and $\mathrm{MH}$ : conduct of the study and manuscript revision. AW: conduct of the study and data acquisition. JA: conduct of the study, manuscript revision and data acquisition.

Funding This study was partially supported by a grant from Boston Scientific (Investigator Sponsored Research).

Competing interests HE has received honoraria for lectures from Bayer, Novartis, Pfizer, Boehringer Ingelheim and Boston Scientific. This study was partially supported by a grant from Boston Scientific (Investigator Sponsored Research).
Patient consent for publication Not required.

Provenance and peer review Not commissioned; internally peer reviewed.

Data availability statement Data are available on reasonable request.

Open access This is an open access article distributed in accordance with the Creative Commons Attribution Non Commercial (CC BY-NC 4.0) license, which permits others to distribute, remix, adapt, build upon this work non-commercially, and license their derivative works on different terms, provided the original work is properly cited, appropriate credit is given, any changes made indicated, and the use is non-commercial. See: http://creativecommons.org/licenses/by-nc/4.0/.

\section{REFERENCES}

1. Chugh SS, Havmoeller R, Narayanan K, et al. Worldwide epidemiology of atrial fibrillation: a global burden of disease 2010 study. Circulation 2014;129:837-47.

2. Björck S, Palaszewski B, Friberg L, et al. Atrial fibrillation, stroke risk, and warfarin therapy revisited: a population-based study. Stroke 2013;44:3103-8.

3. Haim M, Hoshen M, Reges O, et al. Prospective national study of the prevalence, incidence, management and outcome of a large contemporary cohort of patients with incident non-valvular atrial fibrillation. J Am Heart Assoc 2015;4:e001486.

4. Pathan F, Hecht H, Narula J, et al. Roles of Transesophageal Echocardiography and Cardiac Computed Tomography for Evaluation of Left Atrial Thrombus and Associated Pathology: A Review and Critical Analysis. JACC Cardiovasc Imaging 2018;11:616-27.

5. von der Recke G, Schmidt H, Illien S, et al. Transesophageal contrast echocardiography distinguishes a left atrial appendage thrombus from spontaneous echo contrast. Echocardiography 2002;19:343-4.

6. Faez T, Emmer M, Kooiman K, et al. 20 years of ultrasound contrast agent modeling. IEEE Trans Ultrason Ferroelectr Freq Control 2013;60:7-20.

7. Mulvagh SL, DeMaria AN, Feinstein SB, et al. Contrast echocardiography: current and future applications. J Am Soc Echocardiogr 2000;13:331-42.

8. Jung $\mathrm{PH}$, Mueller M, Schuhmann C, et al. Contrast enhanced transesophageal echocardiography in patients with atrial fibrillation referred to electrical cardioversion improves atrial thrombus detection and may reduce associated thromboembolic events. Cardiovasc Ultrasound 2013;11:1.

9. Bernier M, Abdelmoneim SS, Stuart Moir W, et al. CUTE-CV: a prospective study of enhanced left atrial appendage visualization with microbubble contrast agent use during transesophageal echocardiography guided cardioversion. Echocardiography 2013;30:n/a-7.

10. Yao SS, Ilercil A, Meisner JS, et al. Improved Doppler echocardiographic assessment of the left atrial appendage by peripheral vein injection of sonicated albumin microbubbles. Am Heart J 1997;133:400-5.

11. Abdelmoneim SS, Mulvagh SL. Techniques to improve left atrial appendage imaging. J Atr Fibrillation 2014;7:1059.

12. Kirchhof P, Benussi S, Kotecha D, et al. 2016 ESC guidelines for the management of atrial fibrillation developed in collaboration with EACTS. Rev Esp Cardiol 2017;70.

13. von der Recke G, Schmidt H, Illien S, et al. Use of transesophageal contrast echocardiography for excluding left atrial appendage thrombi in patients with atrial fibrillation before cardioversion. J Am Soc Echocardiogr 2002;15:1256-61.

14. Kato $\mathrm{H}$, Nakanishi M, Maekawa $\mathrm{N}$, et al. Evaluation of left atrial appendage stasis in patients with atrial fibrillation using transesophageal echocardiography with an intravenous albumincontrast agent. Am J Cardiol 1996;78:365-9. 